\title{
A!
}

This is an electronic reprint of the original article.

This reprint may differ from the original in pagination and typographic detail.

Ovaska, Markus; Lehtinen, Arttu; Alava, Mikko J.; Laurson, Lasse; Zapperi, Stefano

\section{Excitation Spectra in Crystal Plasticity}

Published in:

Physical Review Letters

DOI:

10.1103/PhysRevLett.119.265501

Published: 27/12/2017

Document Version

Publisher's PDF, also known as Version of record

Please cite the original version:

Ovaska, M., Lehtinen, A., Alava, M. J., Laurson, L., \& Zapperi, S. (2017). Excitation Spectra in Crystal Plasticity. Physical Review Letters, 119(26), 1-5. [265501]. https://doi.org/10.1103/PhysRevLett.119.265501

This material is protected by copyright and other intellectual property rights, and duplication or sale of all or part of any of the repository collections is not permitted, except that material may be duplicated by you for your research use or educational purposes in electronic or print form. You must obtain permission for any other use. Electronic or print copies may not be offered, whether for sale or otherwise to anyone who is not an authorised user. 


\title{
Excitation Spectra in Crystal Plasticity
}

\author{
Markus Ovaska, ${ }^{1}$ Arttu Lehtinen, ${ }^{1}$ Mikko J. Alava, ${ }^{1}$ Lasse Laurson, ${ }^{1}$ and Stefano Zapperi ${ }^{1,2,3}$ \\ ${ }^{1}$ COMP Centre of Excellence, Department of Applied Physics, Aalto University, \\ P.O. Box 11100, FI-00076 Aalto, Espoo, Finland \\ ${ }^{2}$ Center for Complexity and Biosystems, Department of Physics, University of Milano, \\ via Celoria 16, 20133 Milano, Italy \\ ${ }^{3}$ CNR-Consiglio Nazionale delle Ricerche, Istituto di Chimica della Materia Condensata e di Tecnologie per l'Energia, \\ Via R. Cozzi 53, 20125 Milano, Italy \\ (Received 30 August 2017; published 27 December 2017)
}

\begin{abstract}
Plastically deforming crystals exhibit scale-free fluctuations that are similar to those observed in driven disordered elastic systems close to depinning, but the nature of the yielding critical point is still debated. Here, we study the marginal stability of ensembles of dislocations and compute their excitation spectrum in two and three dimensions. Our results show the presence of a singularity in the distribution of excitation stresses, i.e., the stress needed to make a localized region unstable, that is remarkably similar to the one measured in amorphous plasticity and spin glasses. These results allow us to understand recent observations of extended criticality in bursty crystal plasticity and explain how they originate from the presence of a pseudogap in the excitation spectrum.
\end{abstract}

DOI: 10.1103/PhysRevLett.119.265501

Recent advances in crystal plasticity have revealed the importance of collective dislocation dynamics in crystals [1-3]. The key phenomena include power-law distributed strain bursts [4-7] and intermittent acoustic emission signals [8-10], originating from avalanches of dislocation activity. The theoretical description of such crackling noise response in plasticity has been debated, and ideas from depinning transitions [11,12], jamming [13-15], and glassy dynamics [16] of the dislocation assembly have been brought up.

To resolve the question of the fundamental nature of bursty dislocation dynamics, it is central to understand how such activity bursts or excitations are triggered by applied stresses. For amorphous plasticity, this has recently been addressed by considering the pointwise distribution $P(x)$ of the local distances $x$ in stress to a threshold above which a deformation burst is excited [17-19]. In the $x \rightarrow 0$ limit, the form of $P(x)$ encodes information about the nature of the dynamics exhibited by the system, and is connected with the properties of the ensuing crackling noise [20]. One expects a power-law form $P(x) \propto x^{\theta}$, where the exponent $\theta$ characterizes the system. This approach stems from the classical depinning problem of elastic (a convex interaction kernel) interfaces in random media where $P(x)$ is flat for small $x$, so $\theta=0$. In models of amorphous plasticity as well as certain spin glass models, singular behavior of $P(x)$ for $x \rightarrow 0$, or $\theta>0$, is found in the quasistatic limit [17-21]: a "pseudogap" in the excitation spectrum [20].

It has been argued that the singular $P(x)$ is a consequence of the nonpositive definite nature of the long-range interaction kernel mediating the collective deformation dynamics [17,18]. This feature has been linked to the emergence of "extended criticality" in the crackling dynamics, when critical fluctuations take place over an extended range of control parameter values rather than only in the proximity of a "critical point" [20]. In the case of plastic deformation of a system with $N$ dislocations the argument states that if $P(x) \propto x^{\theta}$, the stress increment separating avalanches scales as $N^{-1 /(1+\theta)}$, and hence the number of events $N_{a}$ within a stress interval $\Delta \sigma \sim 1$ scales as $N_{a} \sim N^{1 /(1+\theta)} \ll N$. However, these few events must be responsible for an extensive strain increment, such that the mean avalanche size scales as $N / N_{a} \sim N^{\theta /(1+\theta)}$; i.e., it diverges in the thermodynamic limit. This behavior has been observed in various glassy systems ranging from mean field spin glasses [22,23] to models of amorphous plasticity [21]. Dislocation systems exhibit slow, glasslike dynamics, argued to originate from the frustrated dislocation interactions [16]. Together with recent results of extended criticality $[13,14,24]$ this suggests the possibility of interesting, nontrivial excitation spectra.

In this Letter, we report an extensive study of the excitation spectra in crystal plasticity by considering 2D and 3D discrete dislocation dynamics (DDD) systems. We perform simulations with and without the presence of quenched pinning centers (e.g., solute atoms). The key findings are that the distributions $P(x)$ are singular in pure systems and develop a small $x$ cutoff with quenched disorder. In all cases considered, the exponent $\theta$ is found to depend on dimension, the stress rate of the local perturbation, the presence or absence of quenched pinning, and whether the other dislocations not directly subject to the perturbation are allowed to move or not.

The 2D and 3D DDD models we consider are similar to the standard ones discussed in the literature. The 2D case is 
studied in more depth as the 3D one is intrinsically hard numerically. The starting point in both cases is a relaxed, zero-stress state, which we expect to be in the extended criticality regime of the pure dislocation systems $[13,14,24]$. The 2D DDD model represents a cross section (xy plane) of a single crystal, with a single slip geometry, and straight parallel edge dislocations along the $z$ axis. The $N_{d}$ edge dislocations glide along directions parallel to their Burgers vectors $\mathbf{b}= \pm b \mathbf{u}_{x}$, where $b$ is the magnitude and $\mathbf{u}_{x}$ is the unit vector along the $x$ axis. Equal numbers of dislocations with positive and negative Burgers vectors are assumed, and dislocation climb is not considered. The dislocations interact with each other through their longrange stress fields $\sigma_{x y}=D b x\left(x^{2}-y^{2}\right) /\left(x^{2}+y^{2}\right)^{2}$, where $D=\mu / 2 \pi(1-\nu), \mu$ is the shear modulus, and $\nu$ the Poisson ratio. Dislocation annihilation taking place in real crystals is modeled by removing dislocation pairs with opposite Burgers vectors from the system if their mutual distance is smaller than $b$. In addition, to test the effect of quenched disorder on the excitation spectrum, we perform a set of simulations including also $N_{s}$ randomly positioned immobile pinning centers ("solute atoms") interacting with the dislocations via short-range interactions, employing a regularized interaction energy $U=A s_{n} \sin \theta / r\{1-$ $\left.\exp \left[-\left(k^{2} / a^{2}\right) r^{2}\right]\right\}$, with $k=1.65, a$ the atomic distance, $s_{n}$ the sign of the Burgers vector of the dislocation, and $A=(1+\nu) \mu b \Delta V / 3 \pi(1-\nu)$ an interaction strength parameter, with $\Delta V$ the misfit area [25]. These pinning centers generate a short-range correlated random pinning field [with a correlation length $\xi \approx\left(N_{s} / L^{2}\right)^{-1 / 2}$ and strength proportional to $A$ ] interfering with the dislocation motion [11]. Dislocation dynamics is taken to be overdamped, such that the dislocation velocity is proportional to the Peach-Koehler force due to the total stress (with contributions from dislocation interactions, the random pinning field, as well as from the external stress $\sigma_{\text {ext }}$ when present) acting on it.

The 3D DDD simulations are performed using the ParaDis code [26], considering the fcc crystal structure with material parameters of $\mathrm{Al}$ (shear modulus $G=26 \mathrm{GPa}$, Poisson ratio 0.35 , Young modulus $70.2 \mathrm{GPa}$, Burgers vector $b=2.863 \times 10^{-10} \mathrm{~m}$, and dislocation mobility $10^{4} \mathrm{~Pa}^{-1} \mathrm{~s}$; for simplicity, both edge and screw segments are taken to have the same mobility). The line dislocations are modeled using a nodal discretization scheme where dislocation lines are represented by nodal points connected to their neighbors by straight segments. Changes in dislocation geometry are modeled by adding and removing these nodal points. The total stress acting on a node consists of the external part, resulting from the deformation of the whole crystal, and of the internal, anisotropic stress fields generated by the other dislocations within the crystal. This leads to a Peach-Koehler force moving the discretization nodes according to a materialspecific mobility function which relates the total forces experienced by dislocations to their velocities, encoding also the constraints on dislocation motion due to crystal structure. Forces between segments of nearby nodes and self-interaction of dislocations are calculated with explicit line integrals, while the far-field forces are computed from the coarse-grained dislocation structure using a multipole expansion. In addition, near the dislocation core, local interactions, such as junction formation, annihilation, etc., are introduced phenomenologically with input from smaller scale simulation methods (e.g., MD) and experimental results. A trapezoidal integrator is then used to solve the equation of motion for the discretization nodes. We consider periodic boundary conditions for both 2D and 3D simulations. In each case, a random dislocation configuration (with $N_{d}=400$ in 2D and 40 dislocation lines in 3D) is first let to relax in the absence of applied stresses; after the relaxation, 200 dislocations remain in 2D after the annihilations during relaxation, and the initially straight dislocation lines in 3D develop some curvature and form junctions. We then proceed to study the stability of these relaxed dislocation configurations.

To create excitations in dislocation systems by local stress perturbations, we ramp-up a local external stress $\sigma$ from zero at a constant rate $\sigma_{t}$, and monitor the response of the system by considering the resulting time-dependent global strain rate $\epsilon_{t}$. In the 2D simulations, we go through each individual dislocation one by one, and apply a positive scalar $\sigma=\sigma_{x y}$ only to that [see Fig. 1(a)]. In the 3D case, we choose to apply a local tensile stress within a spherical region, with an example shown in Fig. 1(c) as the red sphere; we consider spheres with radii comparable to the average dislocation spacing $1 / \sqrt{\rho}$, where $\rho$ is the dislocation density, and go through all such nonoverlapping spheres in each system. This particular protocol is mandated by the procedure by which line dislocations are treated in a DDD code such as ParaDis. For both 2D and $3 \mathrm{D}$, an excitation is defined when $\epsilon_{t}$ first exceeds the threshold at a stress $\sigma=\sigma_{\text {ex }}$; see Fig. 1(b). The results are averaged over both different relaxed dislocation configurations (500 and 100 in 2D and 3D, respectively) as well as over different excitation locations in each configuration, i.e., 200 dislocations and 400 spheres per system, resulting in approximately $10^{5}$ and $4 \times 10^{4}$ local stress-strain rate curves in $2 \mathrm{D}$ and $3 \mathrm{D}$, respectively. In $3 \mathrm{D}$, not all of these lead to an excitation due to the tensile applied stress not always producing the relevant resolved shear stress(es) within the excitation volume.

In the 2D case, the $P\left(\sigma_{\mathrm{ex}}\right)$ 's are summarized in Figs. 2 and 3. The $P\left(\sigma_{\mathrm{ex}}\right)$ 's for three different stress rates $\sigma_{t}$ for the "pure" dislocation system are shown in Fig. 2(a). For small $\sigma_{\mathrm{ex}}$, a clear power-law form $P\left(\sigma_{\mathrm{ex}}\right) \propto \sigma_{\mathrm{ex}}^{\theta}$ is observed, indicating singular behavior of $P\left(\sigma_{\mathrm{ex}}\right)$ for $\sigma_{\mathrm{ex}} \rightarrow 0$, with the exponent $\theta$ depending on the stress rate; $\theta$ evolves from $\theta \approx 2.6$ to $\theta \approx 1.6$ as the stress rate is lowered from $\sigma_{t}=$ $2.5 \times 10^{-4}$ to $1 \times 10^{-5}$. Notice that rate-dependent $\theta$ 
(a)

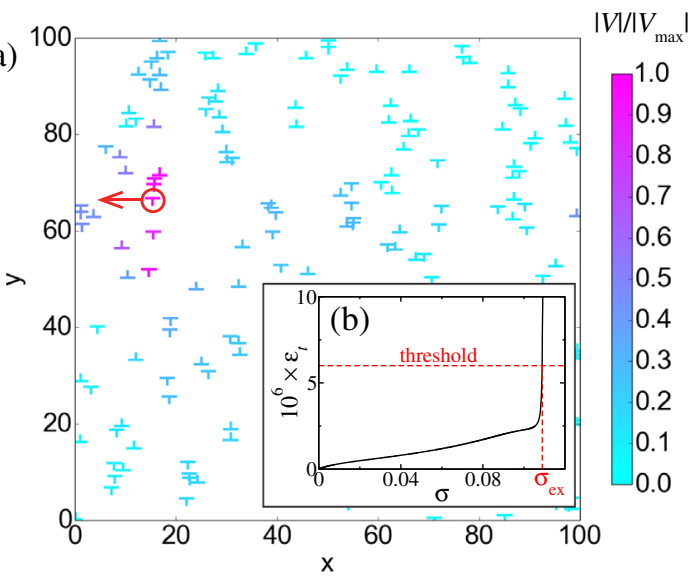

(c)

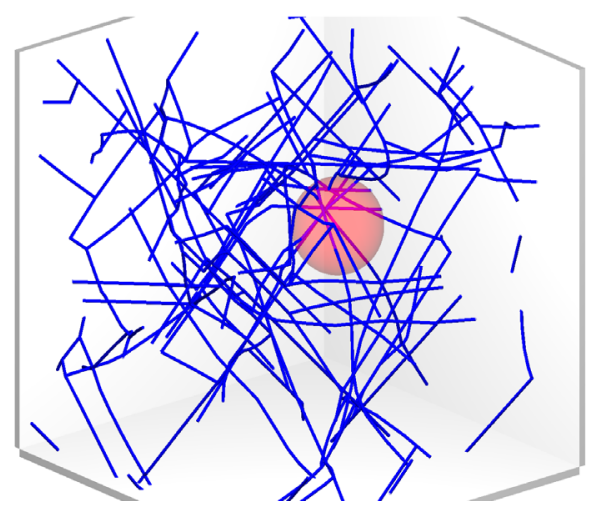

FIG. 1. Idea of the 2D and 3D DDD simulations used to probe the stability of dislocation assemblies against local stress perturbations. (a) Example from a 2D DDD simulation, where a single dislocation (surrounded by a small circle) is subject to a linearly increasing local external stress $\sigma$ (indicated by the arrow), with the color code corresponding to the instantaneous velocity of the dislocations, showing that the dislocation activity tends to spread beyond the dislocation directly subject to the perturbation. (b) Example of the strain rate versus stress graph, along with an illustration of the procedure to define an excitation by thresholding the strain rate signal. (c) Example of the 3D dislocation system, with the red sphere corresponding to the location where the local external stress $\sigma$ is being applied to excite dislocation activity.

exponents have been observed also in elastoplastic models for amorphous materials [19]. It is worth pointing out that in the case considered in Fig. 2(a), all the dislocations are able to respond to the local stress perturbation via the longrange dislocation-dislocation interactions, and thus it sometimes happens that the dislocation activity induced by the local stress is not localized in the immediate vicinity of the dislocation subject to the perturbation; this is illustrated by Movie 1 (Supplemental Material [27]). For comparison, Fig. 2(b) shows the corresponding $P\left(\sigma_{\mathrm{ex}}\right)$ 's in a system where all the other dislocations, except for the one directly subject to the stress perturbation, are kept fixed, in analogy to the procedure employed when considering various lattice models of amorphous plasticity [18]. While the $P\left(\sigma_{\mathrm{ex}}\right)$ 's in
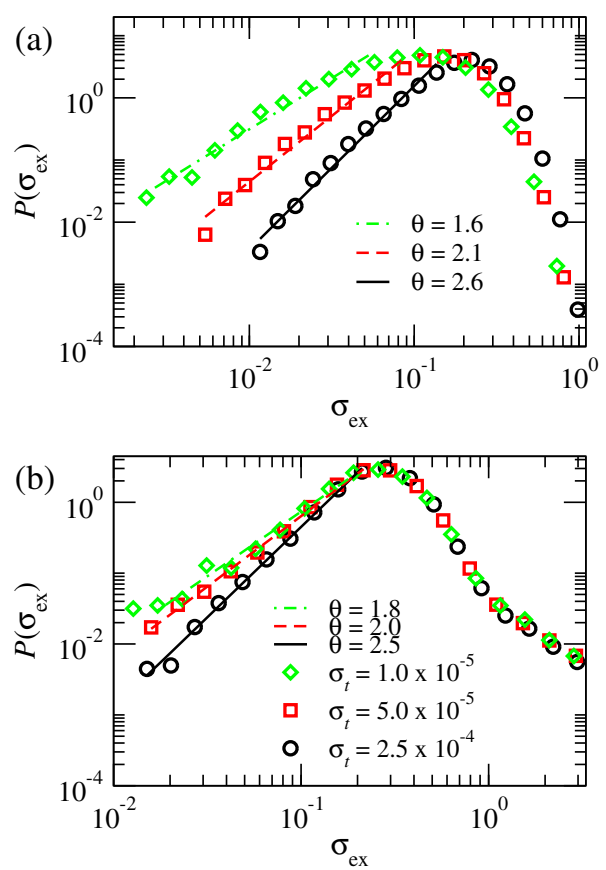

FIG. 2. Excitation spectra $P\left(\sigma_{\mathrm{ex}}\right)$ from 2D DDD simulations for three different stress rates $\sigma_{t}$ (indicated in the legend) for pure dislocation systems. (a) $P\left(\sigma_{\mathrm{ex}}\right)$ for systems where all the dislocations are allowed to move, while in (b) the corresponding data for systems where only the dislocation subject to the local stress perturbation is mobile are displayed. All distributions are singular $(\theta>0)$ for small $\sigma_{\mathrm{ex}}$, with the lines corresponding to fits of the form of $P\left(\sigma_{\mathrm{ex}}\right) \propto \sigma_{\mathrm{ex}}^{\theta}$.

Fig. 2(b) look qualitatively somewhat different from those shown in Fig. 2(a), showing in particular a weaker overall dependence on the stress rate $\sigma_{t}$, the $\sigma_{t}$-dependent exponent $\theta$ nevertheless assumes similar values in both cases: even the single-dislocation excitations display singular characteristics. In all these cases the spectra show that creating excitations becomes harder with higher stress rates: a larger $\sigma_{\mathrm{ex}}$ is typically required for a larger $\sigma_{t}$.

In the presence of quenched disorder, the behavior described above for the pure dislocation systems should change. We show in Fig. 3(a) the $P\left(\sigma_{\mathrm{ex}}\right)$ 's for a system with a quenched pinning field generated by randomly distributed pinning centers of "intermediate" strength, with $A=0.1$ and $N_{s} / L^{2}=0.8 b^{-2}$ chosen to transform the glasslike jamming scenario of pure dislocation systems into a depinninglike problem, but the disorder is not so strong that it would eliminate the scale-free nature of the avalanches (see Ref. [11] for details); we consider the same three stress rates as above to apply the local perturbation. One may make two main observations: (i) the exponent $\theta$ assumes lower stress-rate-dependent values as compared to the pure dislocation system and (ii) the power laws exhibited by the $P\left(\sigma_{\mathrm{ex}}\right)$ 's appear to have low- $\sigma_{\mathrm{ex}}$ cutoffs; hence, we fit the data with the function $P\left(\sigma_{\mathrm{ex}}\right) \propto$ $\sigma_{\mathrm{ex}}^{\theta} \exp \left[-\left(\sigma_{0} / \sigma_{\mathrm{ex}}\right)^{\alpha}\right]$, where $\sigma_{0}$ is the cutoff stress scale. 

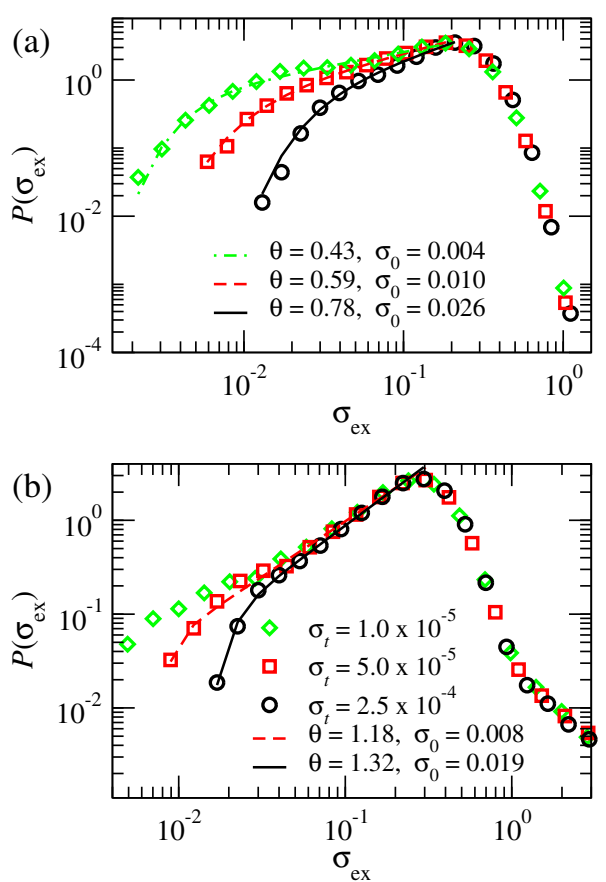

FIG. 3. Excitation spectra $P\left(\sigma_{\mathrm{ex}}\right)$ from 2D DDD simulations for three different $\sigma_{t}$ for dislocation systems containing quenched disorder of "intermediate" strength, inducing depinninglike dislocation dynamics. In (a), all the dislocations are mobile, while in (b), only the dislocation subject to $\sigma$ is allowed to move. The distributions are singular for small $\sigma_{\mathrm{ex}}$, down to a small- $\sigma_{\mathrm{ex}}$ cutoff at $\sigma_{0}$ due to quenched disorder. Lines are fits of the form of $P\left(\sigma_{\mathrm{ex}}\right) \propto$ $\sigma_{\mathrm{ex}}^{\theta} \exp \left[-\left(\sigma_{0} / \sigma_{\mathrm{ex}}\right)^{\alpha}\right]$.

These cutoffs are related to the finite stress needed to push the dislocation out of the local potential energy minimum due to the quenched pinning field; thus, we expect $\sigma_{0}$ to increase with increasing disorder strength. For a given disorder strength, $\sigma_{0}$ is found to increase with increasing $\sigma_{t}$. Notice that no such feature is observable in the pure dislocation system, where dislocation activity may in some instances be triggered with a very small $\sigma_{\mathrm{ex}}$. Figure 3(b) shows the results from the 2D system with pinning and all the other dislocations fixed; again, a small- $\sigma_{\mathrm{ex}}$ cutoff can be observed, and the distributions show less sensitivity to $\sigma_{t}$ than in the case where all dislocations are able to respond to the perturbation.

Finally, we consider the excitations in the 3D DDD simulations, considering for simplicity and due to the high computational cost of the 3D simulations only the case where all dislocations are mobile, and no quenched disorder is present; see Movie 2 in the Supplemental Material [27] for an example of the excitation process. Figure 4 shows that the results are qualitatively similar to those found above for the $2 \mathrm{D}$ system: the distributions $P\left(\sigma_{\mathrm{ex}}\right)$ are singular at $\sigma_{\mathrm{ex}} \rightarrow 0$, and the exponent $\theta$ exhibits a similar dependence on $\sigma_{t}$ as that shown in Fig. 3 for the corresponding 2D system, with $\theta$ varying between $\sim 2.2$ and $\sim 1.4$ for the data shown in Fig. 4. The inset shows the

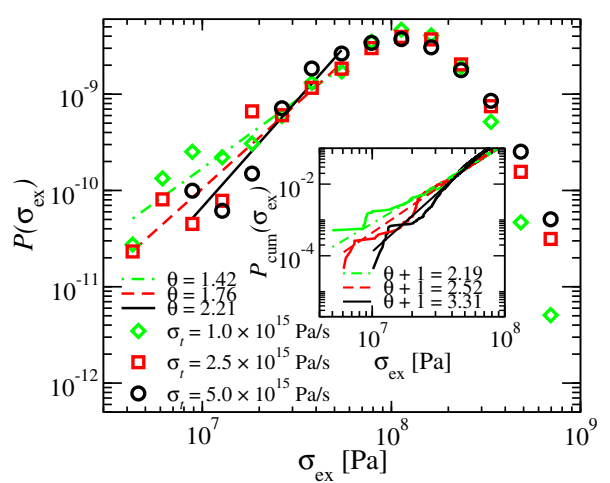

FIG. 4. Excitation spectra $P\left(\sigma_{\text {ex }}\right)$ from 3D DDD simulations for three different $\sigma_{t}$. All dislocations are allowed to move, and there is no quenched disorder. The $\sigma_{t}$-dependent $\theta$ exponents are similar to those found in the corresponding 2D DDD model, and may be approximately recovered also by considering the cumulative distribution function $P_{\mathrm{cum}}\left(\sigma_{\mathrm{ex}}\right)$ (inset).

corresponding cumulative distribution function $P_{\text {cum }}\left(\sigma_{\mathrm{ex}}\right)$ for small $\sigma_{\mathrm{ex}}$, exhibiting scaling with an exponent $\theta+1$, with the $\theta$ values in reasonable agreement with those estimated from the $P\left(\sigma_{\mathrm{ex}}\right)$ 's. Considering different threshold values for the strain rate to define the excitations leads to slightly threshold-dependent exponents in the 3D case (not shown), but the qualitative picture remains the same for a range of threshold values. Thus, a "pseudogap" appears to be present in the excitation spectrum also in 3D dislocation systems.

To summarize, we have performed both 2D and 3D DDD simulations to establish that the excitation spectra in crystal plasticity exhibit singular behavior at $\sigma_{\mathrm{ex}} \rightarrow 0$, something that is at odds with the known behavior of elastic manifolds in random media exhibiting a depinning transition. As this behavior persists when moving from $2 \mathrm{D}$ systems to $3 \mathrm{D}$ ones, the root cause is shared by both systems. The likely cause is the anisotropic, nonpositive definite interactions between dislocations, in analogy to the quadrupolar Eshelby-type stress fields argued to be responsible for the similar behavior found recently for amorphous plasticity $[17,18]$. This then persists upon coarse graining, and manifests itself in the presence of critical-like fluctuations or plastic avalanches even at negligible external stresses $[13,14,24]$. The presence of a frozen impurity field, on the other hand, leads to a finite minimum for the excitation stress $\sigma_{\mathrm{ex}}$, in agreement with the idea of the presence of a true critical point or yield stress [11] instead of the extended criticality scenario of pure dislocation systems. Finally, we point out that 2D colloidal crystals [28] might provide an interesting experimental system to test our results.

This work has been supported by the Academy of Finland through its Centres of Excellence Programme (2012-2017) under Project No. 251748, an Academy Research Fellowship (L.L., Project No. 268302), and the FiDiPro program (S.Z., Project No. 13282993). We 
acknowledge the computational resources provided by the Aalto University School of Science "Science-IT" project, as well as those provided by CSC (Finland). S.Z. is supported by the ERC Advanced Grant No. 291002 SIZEFFECTS.

[1] M. Zaiser, Adv. Phys. 55, 185 (2006).

[2] M. J. Alava, L. Laurson, and S. Zapperi, Eur. Phys. J. Spec. Top. 223, 2353 (2014).

[3] G. Ananthakrishna, Phys. Rep. 440, 113 (2007).

[4] M. D. Uchic, P. A. Shade, and D. M. Dimiduk, Annu. Rev. Mater. Res. 39, 361 (2009).

[5] D. M. Dimiduk, C. Woodward, R. LeSar, and M. D. Uchic, Science 312, 1188 (2006).

[6] K. Ng and A. Ngan, Acta Mater. 56, 1712 (2008).

[7] M. Zaiser, J. Schwerdtfeger, A. Schneider, C. Frick, B. G. Clark, P. Gruber, and E. Arzt, Philos. Mag. 88, 3861 (2008).

[8] J. Weiss, W. B. Rhouma, T. Richeton, S. Dechanel, F. Louchet, and L. Truskinovsky, Phys. Rev. Lett. 114, 105504 (2015).

[9] J. Weiss and J.-R. Grasso, J. Phys. Chem. B 101, 6113 (1997).

[10] M.-C. Miguel, A. Vespignani, S. Zapperi, J. Weiss, and J.-R. Grasso, Nature (London) 410, 667 (2001).

[11] M. Ovaska, L. Laurson, and M. J. Alava, Sci. Rep. 5 (2015).

[12] N. Friedman, A. T. Jennings, G. Tsekenis, J.-Y. Kim, M. Tao, J. T. Uhl, J. R. Greer, and K. A. Dahmen, Phys. Rev. Lett. 109, 095507 (2012).

[13] P. D. Ispánovity, L. Laurson, M. Zaiser, I. Groma, S. Zapperi, and M. J. Alava, Phys. Rev. Lett. 112, 235501 (2014).
[14] A. Lehtinen, G. Costantini, M. J. Alava, S. Zapperi, and L. Laurson, Phys. Rev. B 94, 064101 (2016).

[15] M.-Carmen Miguel, A. Vespignani, M. Zaiser, and S. Zapperi, Phys. Rev. Lett. 89, 165501 (2002).

[16] B. Bakó, I. Groma, G. Györgyi, and G. T. Zimányi, Phys. Rev. Lett. 98, 075701 (2007).

[17] J. Lin, A. Saade, E. Lerner, A. Rosso, and M. Wyart, Europhys. Lett. 105, 26003 (2014).

[18] J. Lin, E. Lerner, A. Rosso, and M. Wyart, Proc. Natl. Acad. Sci. U.S.A. 111, 14382 (2014).

[19] C. Liu, E. E. Ferrero, F. Puosi, J.-L. Barrat, and K. Martens, Phys. Rev. Lett. 116, 065501 (2016).

[20] M. Müller and M. Wyart, Annu. Rev. Condens. Matter Phys. 6, 177 (2015).

[21] J. Lin, T. Gueudré, A. Rosso, and M. Wyart, Phys. Rev. Lett. 115, 168001 (2015).

[22] F. Pázmándi, G. Zaránd, and G. T. Zimányi, Phys. Rev. Lett. 83, 1034 (1999).

[23] L. Yan, M. Baity-Jesi, M. Müller, and M. Wyart, Phys. Rev. Lett. 114, 247208 (2015).

[24] S. Janićević, M. Ovaska, M. J. Alava, and L. Laurson, J. Stat. Mech. (2015) P07016.

[25] R. Wang, J. Phys. D 23, 263 (1990).

[26] A. Arsenlis, W. Cai, M. Tang, M. Rhee, T. Oppelstrup, G. Hommes, T. G. Pierce, and V. V. Bulatov, Model. Simul. Mater. Sci. Eng. 15, 553 (2007).

[27] See Supplemental Material at http://link.aps.org/ supplemental/10.1103/PhysRevLett.119.265501 for movies illustrating the way in which dislocation activity is excited by applied local stress perturbations.

[28] A. Pertsinidis and X. S. Ling, New J. Phys. 7, 33 (2005). 